\title{
Estimation of the Regularisation Parameter in Huber-MRF for Image Resolution Enhancement
}

\author{
Sakinah Ali Pitchay ${ }^{1}$ and Ata Kabán ${ }^{2}$ \\ ${ }^{1}$ Faculty of Science and Technology, Universiti Sains Islam Malaysia (USIM), \\ Bandar Baru Nilai, 71800 Nilai, Negeri Sembilan, Malaysia \\ 2 School of Computer Science, University of Birmingham, \\ Edgbaston, Birmingham, B15 2TT, UK \\ \{S.A.Pitchay,A.Kaban\}@cs.bham.ac.uk, sakinah.ali@usim.edu.my
}

\begin{abstract}
The Huber Markov Random Field (H-MRF) has been proposed for image resolution enhancement as a preferable alternative to Gaussian Random Markov Fields (G-MRF) for its ability to preserve discontinuities in the image. However, its performance relies on a good choice of a regularisation parameter. While automating this choice has been successfully tackled for G-MRF, the more sophisticated form of HMRF makes this problem less straightforward. In this paper we develop an approximate solution to this problem, by upper-bounding the partition function of the H-MRF. We demonstrate the working and flexibility of our approach in image super-resolution experiments.
\end{abstract}

Keywords: Huber prior, Hyper-parameter Optimisation, Superresolution.

\section{Introduction}

Image super-resolution seeks to enhance the resolution of an image beyond of that of the detector arrays of the cameras, by appropriately combining together the available low resolution measurements. The difficulty lies with the ill-posed nature of this problem, which often requires the solution of a severely underdetermined system. The use of an appropriate image model, or prior to regularise the problem is therefore crucial. To this end, Gaussian Markov Random Fields (G-MRF) represents a convenient common choice which, however is known to have a tendency to blur the edges of the super-resolved image 447. HuberMarkov Random Fields (H-MRF) have been designed to address this problem, and represent a state of the art approach for super-resolution 477. The Huber function has longer tails than the Gaussian, hence the edges of the image get less penalised. In addition, the Huber prior is convex; hence, if coupled with a Gaussian observation model, it guarantees a unique solution.

However, the good performance of H-MRF relies on a good choice of its free parameters. While automating this choice has been successfully tackled for G-MRF [2], the same is currently not available for H-MRF. Due to the intractable form of the partition function of Huber-MRF, a principled model-based estimation 
of the hyper-parameters has been found problematic [7, and various heuristic approaches have been in use [47] to sidestep the computational burden of crossvalidation. In this paper, we develop an approximation, which allows us to obtain an estimate of the regularisation parameter of the H-MRF prior simultaneously with obtaining the resolution enhancement.

\section{Model}

\subsection{The Observation Model}

We denote the original, high-resolution image by the column vector $\boldsymbol{z}$ throughout the paper. This is a vectorised form of the image, and contains $N$ pixel intensities. The degradation process will be taken as a linear transform that consists of shifts, blur and down-sampling. With $k$ low resolution versions of the original image, $W$ will denote the $k$ linear transforms stacked into a single $M \times N$ matrix, where $M$ is the total number of observed low resolution pixels. Likewise, the $M$-dimensional column vector $\boldsymbol{y}$ will contain the vectorised low resolution observations stacked together. This allows us to write the observation model in a simple form, as follows:

$$
\boldsymbol{y}=\boldsymbol{W} \boldsymbol{z}+\boldsymbol{\eta}
$$

where $\boldsymbol{\eta} \sim \mathcal{N}\left(\mathbf{0}, \sigma^{2} \boldsymbol{I}\right)$ is additive noise.

\subsection{The Huber-MRF Prior}

Huber Markov Random Field (H-MRF) image-prior is studied in [4|5|7] and the univariate Huber density is defined as:

$$
p(u \mid \alpha)=\frac{1}{Z(\alpha)} \exp \{-\alpha g(u)\}
$$

where

$$
g(u \mid T)=\left\{\begin{aligned}
u^{2}, & \text { if }|u|<T ; \\
2 T|u|-T^{2}, & \text { if }|u| \geq T .
\end{aligned}\right.
$$

is the Huber function, $T$ is a threshold parameter, and $Z(\alpha)$ is the partition function, independent of $u$. The Huber function takes a threshold parameter $T$ that specifies the value at which it diverts from being quadratic to being linear. If the threshold $T$ is large, then the H-MRF prior reduces to a Gaussian-MRF image-prior. On the other hand, if $T \rightarrow 0$, the Huber prior is equivalent to an L1-regularisation based prior.

The first order MRF model posits that each pixel intensity depends only on its immediate neighbours. Here we condition on the average of the four cardinal neighbours, following [34, which appears to be a both simple and efficient choice. For convenient notation, the $N \times N$ matrix $\boldsymbol{D}$ is defined with values 1 in its diagonal entries, $d_{i j}=-1 / 4$ whenever $j$ is a cardinal neighbour of $i$, and 0 
everywhere else. $\boldsymbol{D}_{i}$ will denote the $i$-th row of this matrix. Then we can write the difference between the $i$-th pixel and its average neighbours simply as $\boldsymbol{D}_{i} \boldsymbol{z}$. With these notations in place, the $\mathrm{H}-\mathrm{MRF}$ prior is the following:

$$
p(\boldsymbol{z} \mid \alpha)=\frac{1}{Z_{M R F}(\alpha)} \exp \left\{-\alpha \sum_{i=1}^{N} g\left(\boldsymbol{D}_{i} \boldsymbol{z}\right)\right\}
$$

The partition function of the MRF, $Z_{M R F}(\alpha)$, is independent of $\boldsymbol{z}$.

\section{MAP Estimation of $z$}

The most probable high resolution image is given by maximising the log posterior of $\boldsymbol{z}$, that is, up to additive constants, equivalent to minimising the negative log of the joint likelihood:

$$
\begin{aligned}
L(\boldsymbol{z}) & =-\log p(\boldsymbol{y} \mid \boldsymbol{W} \boldsymbol{z}) p(\boldsymbol{z}) \\
& =\frac{1}{2 \sigma^{2}}(y-W z)^{2}+\alpha \sum_{i=1}^{N} g\left(\boldsymbol{D}_{i} \boldsymbol{z}\right)
\end{aligned}
$$

Notice the normalising term $Z(\alpha)$ is constant here, hence it is not needed. This optimisation is then fairly straightforward. Being a convex objective, any nonlinear optimiser will guarantee a global optimum. The gradient of the prior term is:

$$
\frac{\partial-\log p(\boldsymbol{z} \mid \alpha)}{\partial \boldsymbol{z}}=\alpha \sum_{i=1}^{N} g^{\prime}\left(\boldsymbol{D}_{i} \boldsymbol{z}\right)=\alpha \boldsymbol{D} g^{\prime}(\boldsymbol{D} \boldsymbol{z})
$$

where the derivative function $g^{\prime}()$ acts component-wise, and $\boldsymbol{D}$ is the $N \times N$ matrix with rows $\boldsymbol{D}_{i}$. We employed this in a conjugate gradient optimisation procedure.

The high-resolution estimate is then given by the solution of the stationary system of equations:

$$
\boldsymbol{W}^{T}(\boldsymbol{y}-\boldsymbol{W} \boldsymbol{z})+\lambda \boldsymbol{D} g^{\prime}(\boldsymbol{D} \boldsymbol{z})=0
$$

where both $\alpha$ and the variance of the likelihood term are now absorbed in the the parameter $\lambda:=\alpha \sigma^{2}$ may be referred to as the regularisation parameter.

\section{Determining $\lambda$ by Integrating over $\alpha$}

To deal with the hyper-parameter $\alpha$, we may choose to place the Jeffreys prior (termed often as the ignorance prior) on it, and integrate $\alpha$ out from the model. This approach is the so-called Bayesian regularisation. It was first introduced in [8], and subsequently shown to work well for a number of models in the literature. In our case, the Jeffreys prior is:

$$
p(\alpha) \propto \frac{1}{\alpha}
$$


Hence, the marginal prior becomes:

$$
p(\boldsymbol{z})=\int_{0}^{\infty} p(\boldsymbol{z} \mid \alpha) p(\alpha) d \alpha
$$

where the conditional prior $p(\boldsymbol{z} \mid \alpha)$ is the one defined in eq.(4). However, since we have an MRF model, the partition function $Z_{M R F}(\alpha)$ of our conditional prior $p(\boldsymbol{z} \mid \alpha)$ is an intractable multivariate integral.

$$
Z_{M R F}(\alpha)=\int d z \exp \left\{-\alpha \sum_{i=1}^{N} g\left(\boldsymbol{D}_{i} \boldsymbol{z}\right)\right\}
$$

It does not depend on $\boldsymbol{z}$, but it does depend on $\alpha$, - hence we cannot ignore it when we try to estimate both $\alpha$ and $\boldsymbol{z}$ simultaneously. To approximate this integral, we proceed in two steps: we create a reasonably tight upper-bound to the partition function of the univariate Huber density; then we use this in a pseudolikelihood approximation of the MRF partition function.

\subsection{Upper-Bounding $\mathrm{Z}(\alpha)$}

$Z(\alpha)$ may be written with the use of the error function $\operatorname{erf}(x)=\frac{2}{\sqrt{\pi}} \int_{0}^{x} \exp \left(-t^{2}\right) d t$, it is however uneasy to work with this directly. Instead, we create a convenient analytic upper-bound as the following:

$$
\begin{aligned}
Z(\alpha)=\int \exp (-\alpha g(u)) d u & =\frac{\exp \left(-\alpha T^{2}\right)}{\alpha T}+\operatorname{erf}(T \sqrt{\alpha}) \sqrt{\pi / \alpha} \\
& \leq \frac{\exp \left(-\alpha T^{2}\right)}{\alpha T}+\frac{\exp \left(\alpha T^{2}\right)-\exp \left(-\alpha T^{2}\right)}{\alpha T} \\
& =\exp \left(\alpha T^{2}\right) /(\alpha T)
\end{aligned}
$$

Note that, since the upper-bound approximation to $Z(\alpha)$ was made on the interval $[-T, T]$, the bound will be tighter for smaller values of $T$. Fortunately, this is not restrictive in practice, since it was empirically observed $[7$ that the sensible range of values is somewhere in $[0.01,0.1]$. In this range our bound is tight, as shown in Figure 1. The bound also tightens for smaller $\alpha$.

\subsection{Approximating $Z_{M R F}(\alpha)$}

To be able to employ the upper bound derived in the previous section, we shall combine it with the well-known pseudolikelihood approximation on $Z_{M R F}(\alpha)$. Besides its simplicity, the pseudo-likelihood is known to enjoy consistency [1]. It consists of taking each $\boldsymbol{D}_{i} \boldsymbol{z}$ as if it was independent of $\boldsymbol{D}_{j} \boldsymbol{z}$, for all $j \neq i$, to make the problem tractable. This allows us to employ our upper-bound derived earlier, for each variable in turn. Thus, we have the following for (11):

$$
Z_{M R F}(\alpha) \approx C \cdot \exp \left\{T^{2} \alpha\right\}^{N} \alpha^{-N}
$$




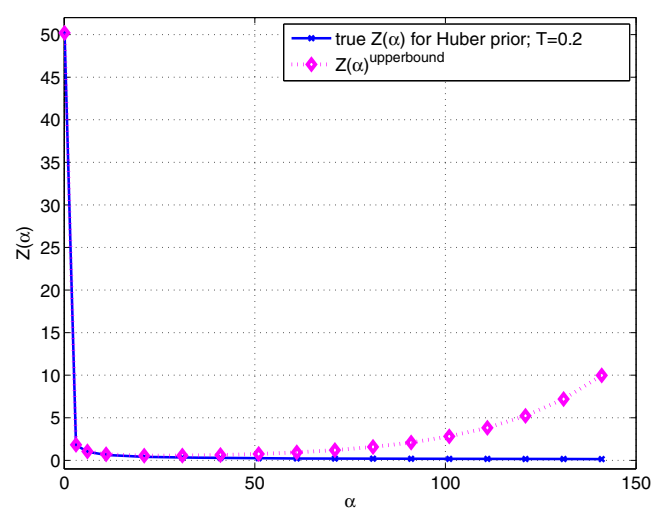

Fig. 1. Tightness of the upper-bound approximation to $Z(\alpha)$, with threshold $T=0.2$. For smaller values of the threshold, the upper-bound gets practically indistinguishable from the true partition function.

where we used the upper bounds (14) on each $u_{i}=\boldsymbol{D}_{i} \boldsymbol{z}$, and $C$ is a constant that subsumes the factors that do not depend on $\alpha$. Having derived a workable approximation to $Z_{M R F}(\alpha)$, we replace this into (10), so the marginalisation over $\alpha$ becomes tractable, and yields the marginal prior:

$$
\begin{aligned}
p(\boldsymbol{z}) & \approx C \cdot \int \alpha^{N-1} \exp \left\{-\alpha\left[T^{2} N+\sum_{i=1}^{N} g\left(\boldsymbol{D}_{i} \boldsymbol{z}\right)\right]\right\} d \alpha \\
& \propto \frac{\Gamma(N)}{\left\{T^{2} N+\sum_{i=1}^{N} g\left(\boldsymbol{D}_{i} \boldsymbol{z}\right)\right\}^{N}} \propto\left\{T^{2} N+\sum_{i=1}^{N} g\left(\boldsymbol{D}_{i} \boldsymbol{z}\right)\right\}^{-N}
\end{aligned}
$$

where we used that $\int_{0}^{\infty} \alpha^{\nu-1} e^{m \alpha} d \alpha=\frac{\Gamma(\nu)}{m^{\nu}}$.

In consequence, the negative log of the marginal prior is:

$$
-\log p(\boldsymbol{z})=N \log \left\{N T^{2}+\sum_{i=1}^{N} g\left(\boldsymbol{D}_{i} \boldsymbol{z}\right)\right\}+\text { const. }
$$

\subsection{Determining $\lambda$}

Now, $\alpha$ is no longer in the model, so we may proceed to optimising $z$, as before, but now using the marginal prior in place of the conditional prior. Taking the gradient from (17) wrt. $\boldsymbol{z}$,

$$
\frac{\partial-\log p(\boldsymbol{z})}{\partial \boldsymbol{z}}=N \frac{1}{N T^{2}+\sum_{i=1}^{N} g\left(\boldsymbol{D}_{i} \boldsymbol{z}\right)} \boldsymbol{D} g^{\prime}(\boldsymbol{D} \boldsymbol{z})
$$

we observe this has a similar form as the gradient we had before from the conditional prior, i.e. eq. (7), except that in place of $\alpha$ now we can isolate the following 
expression that we may consider an estimate of it:

$$
\hat{\alpha}=N \frac{1}{N T^{2}+\sum_{i=1}^{N} g\left(\boldsymbol{D}_{i} \boldsymbol{z}\right)}
$$

Finally, the noise variance is estimated as the following. To avoid singularities, here we use an improper prior $\sigma^{2} \propto \exp \left(-\gamma /\left(2 \sigma^{2}\right)\right)$ to discourage zero values, which gives the estimate:

$$
\hat{\sigma^{2}}=\frac{1}{M}\left(\sum_{i=1}^{M}\left(\boldsymbol{y}_{i}-\boldsymbol{W}_{i} \hat{\boldsymbol{z}}\right)^{2}+\gamma\right)
$$

where $M$ is the total number of pixels in $\boldsymbol{y}$. A small value $\gamma=10^{-12}$ was used in the experiments, to keep the bias minimal.

Putting together (19) and (20), we obtain the following estimate of the regularisation parameter $\lambda$, for use within the stationary equation (7):

$$
\hat{\lambda}=\frac{\frac{1}{M}\left(\sum_{i=1}^{M}\left(\boldsymbol{y}_{i}-\boldsymbol{W}_{i} \hat{\boldsymbol{z}}\right)^{2}+\gamma\right)}{T^{2}+\frac{1}{N} \sum_{i=1}^{N} g\left(\boldsymbol{D}_{i} \hat{\boldsymbol{z}}\right)}
$$

This is our estimate of the regularisation parameter.

Observe that, equation (21) is quite interpretable: $\hat{\lambda}$ is proportional to the noise variance, and inversely proportional to the sum of the squared threshold plus the average smoothness penalty. The threshold parameter $T$ may be understood as controlling the high frequency vs. low frequency components, which can be set within a sensible range e.g. using synthetic data as proposed in [5].

\section{Experiments}

We test our method in severely under-determined systems in the first instance. The original image is the $130 \times 130$ "Butterfly". In each experiment, we compose $\boldsymbol{W}$ from a randomly parameterised homography transform, blur with point spread function (PSF) of standard deviation 0.4, and a specified down-sampling ratio, and then we fix it in order to assess the recovery abilities of the prior. We create three low resolution (LR) versions of the original ground truth, each of size $35 \times 35$ pixels, and we add a small amount of noise to these $\left(\sigma=2.55 \times 10^{-4}\right.$ grey levels). That is, from $M=1225$ observed LR pixels we try to recover $N$ $=16900$ (i.e. c.c.a. 4.5 times more) HR ones. Figure 2 shows the results. The averaged bicubic interpolation is given as the strawmans baseline. However, we see that our adaptive method of estimating $\lambda$ is preferable to using a fixed $\lambda$ in H-MRF, despite the value $\lambda=0.01$ should be a sensible one, based on the results in 7. Our estimate of $\lambda$ is of the order of $10^{-12}$.

In the above experiments, the threshold parameter $T=0.04$ was used, cf. [6]. To give quantitative figures, and to see how the performance varies with the threshold $T$, the left hand plot of Figure 3 shows the mean square error (MSE) 

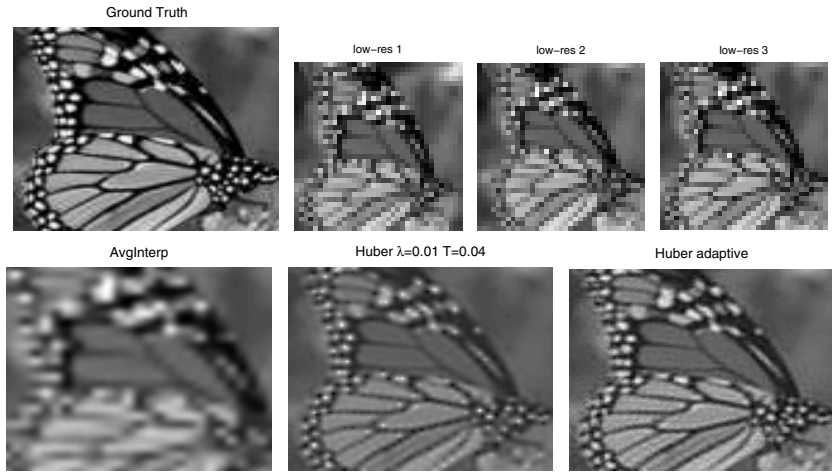

Fig. 2. Top row: The ground truth $(130 \times 130)$ of a butterfly and the 3 low-resolution $(35 \times 35)$ images; Bottom row: Recovered HR images $(130 \times 130)$ comparatively by averaged bicubic interpolation, Huber with fixed $\lambda=0.01$; and Adaptive Huber.
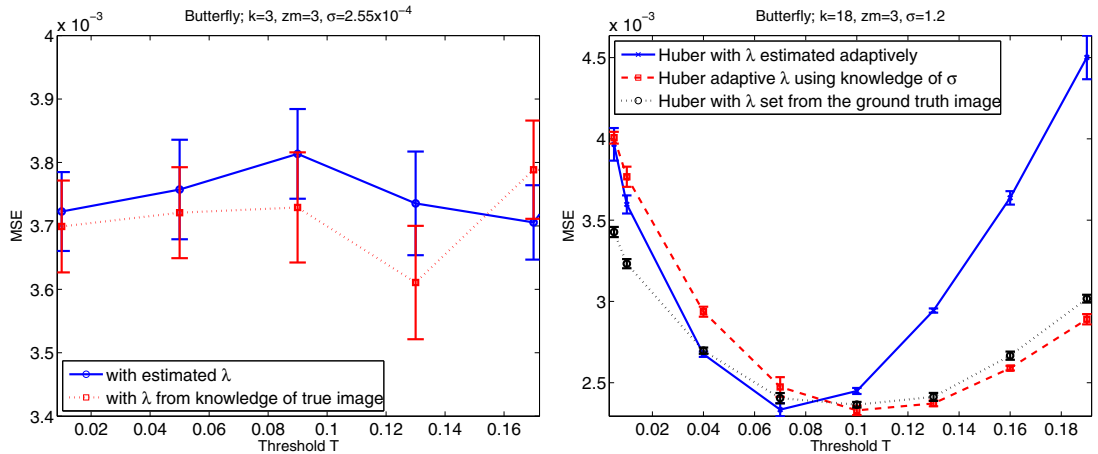

Fig. 3. Dependence on the threshold parameter $T$ and comparison with informed choices of $\lambda$. Left: Under-determined system, small noise $(k=3$ LR images of $35 \times 35$ pixels each, additive noise with $\sigma=2.55 \times 10^{-4}$ grey levels); Right: Overdetermined system ( $k=18 \mathrm{LR}$ observations of $30 \times 30$ pixels each), higher noise condition ( $\sigma=1.2$ grey levels). We see that a larger $T$ is preferred in the latter situation, whereas a range of $T$ works well for the former. We also find that the MSE produced with $\lambda$ estimated adaptively is comparable to that obtained with more informed $\lambda$ settings. The error bars represent one standard error over 10 independent realisations of the system matrix $\boldsymbol{W}$ and the additive noise.

between the recovered and the true image against varying $T$. We see that, there is little variation in this case, most likely because the level of noise is small. On this plot, we also superimposed the MSE figures that we could get if $\lambda$ was set knowing the ground truth image. We see, the obtained MSE values are not very far, in fact comparable to the idealised optimal ones, and the variation with $T$ is also negligible.

The right hand plot repeats the experiment in an overdetermined system and in higher noise conditions. We see, the value of $T$ matters in this case, and there is clearly an optimal range. Three sets of results are superimposed here: with 
$\lambda$ estimated adaptively from the data; with $\lambda$ estimated under assumption that the noise variance is known, and with $\lambda$ estimated from the ground truth image. We can see the MSE performances are comparable in this setting as well.

\section{Conclusions}

We developed a new method for estimating the regularisation parameter for MAP-based resolution enhancement with Huber-MRF priors. This was achieved by upper-bounding the partition function in the Huber density in conjunction with a pseudo-likelihood approach. We demonstrated the added flexibility achieved over the traditional Huber-MRF, and the good performance of our approach in severely under-determined image super-resolution, as well as in overdetermined systems. In future work it will be of interest to compare the adaptive prior presented here with non-convex prior formulations such as [6]. Non-convex priors generally have a better ability to recover texture than convex priors can, but are more difficult to optimise. We should note, despite each iteration of our algorithm solves a convex problem, the marginal prior in eq. (10) is akin a scalemixture, and is non-convex. Therefore the approach presented here may be seen as breaking down a non-convex prior based optimisation problem into a series of convex ones.

\section{References}

1. Geman, S., Gragne, C.: Markov random field image models and their applications to computer vision. In: Proc. Intl Congress of Mathematicians, pp. 1496-1517 (1986)

2. Zibetti, M.V.W., Bazan, F.S.V., Mayer, J.: Determining the regularization parameters for super-resolution problems. Signal Processing 88, 2890-2901 (2008)

3. Hardie, R.C., Barnard, K.J.: Joint MAP Registration and High-Resolution Image Estimation Using a Sequence of Undersampled Images. IEEE Trans. Image Processing 6(12), 621-633 (1997)

4. He, H., Kondi, L.P.: MAP Based Resolution Enhancement of Video Sequences Using a Huber-Markov Random Field Image Prior Model. ICIP (2), 933-936 (2003)

5. He, H., Kondi, L.: Choice of Threshold of the Huber-Markov Prior in Map-based Video Resolution Enhancement. In: Proc. IEEE Canadian Conf. on Electrical and Computer Engineering, Niagara Falls, Canada, vol. II, pp. 801-804 (May 2004)

6. Kabán, A., Ali-Pitchay, S.: Single-frame Image Recovery using a Pearson type VII MRF. Neurocomputing 80, 111-119 (2012)

7. Pickup, L.C., Capel, D.P., Roberts, S.J., Zissermann, A.: Bayesian Methods for Image Super-Resolution. The Computer Journal 52, 101-113 (2009)

8. Buntine, W.L., Weigend, A.S.: Bayesian back-propagation. Complex Systems 5, 603643 (1991) 